\title{
A Compact In-Line Waveguide-to-Microstrip Transition in the Q-Band for Radio Astronomy Applications
}

\author{
Marco Simone ${ }^{1}$, Alessandro Fanti ${ }^{1}$ (i), Giuseppe Valente ${ }^{2}$, Giorgio Montisci ${ }^{1, *(1)}$, \\ Riccardo Ghiani ${ }^{1}$ and Giuseppe Mazzarella ${ }^{1}$ (iD \\ 1 Department of Electrical and Electronic Engineering, University of Cagliari, Piazza D'Armi, \\ 09123 Cagliari, Italy; marco.simone84@gmail.com (M.S.); alessandro.fanti@diee.unica.it (A.F.); \\ ghiani.riccardo@gmail.com (R.G.); mazzarella@diee.unica.it (G.M.) \\ 2 Italian Space Agency, 00133 Rome, Italy; giuseppe.valente@asi.it \\ * Correspondence: giorgio.montisci@unica.it; Tel.: +39-070-675-5780
}

Received: 5 January 2018; Accepted: 16 February 2018; Published: 24 February 2018

\begin{abstract}
A microstrip-to-waveguide transition has been realized for radio astronomy applications, designed to operate in the Q-band (33-50 GHz). As part of an array radio frequency (RF) receiver, the main requirement of such a transition is the reduction of transverse space occupation for the integration in the entire receiver chain, so an in-line configuration has been developed. Moreover, the high frequency band implies that an easy fabrication is a critical requirement if a good match between the two guiding structures is to be obtained in the desired band (with a relative bandwidth of $40 \%$ ). The combination of CAD software and an optimization tool allows the device to achieve a good return loss over the entire band.
\end{abstract}

Keywords: transition; end-launcher; waveguide; microstrip; optimization; PSO

\section{Introduction}

Radioastronomy deals with radio frequency (RF) signals from space. The detection of such waves, with a very low energy, requires large antennas in the microwave and millimeterwave spectrum. Generally speaking, they consist of a parabolic dish and a feed placed in its focus, and this feed collects the waves reflected by the paraboloid. Then, the signal caught by the feed is manipulated by means of a set of devices which form the receiver system.

The heterodyne receiver is a common solution for radioastronomy applications. It converts the RF input into an infrared frequency (IF) input at a lower frequency and keeps the same information [1]. Since the signals caught from the antenna are very low in power, the components of the receiver chain must have low losses and noise [2,3]. Moreover, the high frequencies of certain signals processed in radioastronomy require very small devices, whose constructive tolerances play a critical role in the design.

Multi-feed receivers are usually employed in modern radiotelescopes, since they allow for a quick sweep of the region to be observed. In addition, multiple receptions from the same point of the sky provide a large dataset of detections and allows for the removal of atmospheric interference from the data [4]. A typical feed for an RF receiver is a two-dimensional array of corrugated horn antennas. The horns of the multi-feed system should be as close as possible to the primary focus of the radiotelescope, and close to each other, since their position determines the optical path of the rays reflected from the dish. Horn antennas are easy to construct and are characterized by a high gain and, at high frequencies, low losses. Moreover, they guarantee the wide-band operation required in radioastronomy [5] (around 40\%). The entire front-end of the receiver (basically, horn and low-noise amplifier) works at high frequency and is built using waveguide technology. The part of the system 
that operates at IF is usually realized in planar technology, which provides a more compact solution. The connection between different devices is a crucial point in a transmission system, and appropriate transitions are necessary so that signal information is not altered, and the required bandwidth and a low insertion loss are ensured. The waveguide-to-microstrip transition is the most common interface between horn antennas and planar circuits in microwave and millimeter-wave systems, and it is immediately followed by the mixer. The limitation in space due to the multifeed configuration implies that the typical transition, where the microstrip is inserted in the E-plane of the waveguide to couple the fields, is not a feasible solution. A collinear transition, which keeps a small transverse size of the system, is preferred.

In-line transitions involving waveguides have been investigated since the 1950s, when Wheeler [6] studied the transition from waveguide to a coaxial line with a frequency band of more than $30 \%$. The inner conductor of the cable enters the waveguide, and it is shorted to one of the broad faces of the guide. A step-ridge transformer on the opposite face completes the matching. As regards the waveguide-to-microstrip transition, an in-line configuration was proposed by Yao [7] in 1994, who, in terms of mode matching, performed a full wave modal analysis of the discontinuity between the microstrip and the ridge waveguide. Deslandes [8] presented a particular transition where the waveguide is integrated in the same microstrip dielectric.

Several end-launcher transitions have been investigated by the scientific community. A possible solution consists in the insertion of antennas in the waveguide. In [9], a coaxial-fed circular patch, placed parallel to the waveguide back-short, is presented, with a very compact geometry, but the patch's return loss is only good for part of the X-band. A planar quasi-yagi antenna launcher [10], inserted in the E-plane of the waveguide, with a director element coupled to the microstrip line, provides a quite-flat return loss of $12 \mathrm{~dB}$ on a $40 \%$ bandwidth (in the X-band). However, the geometry on which this planar launcher is based is quite complicated. A bow-tie antenna [11] provides a 36\% bandwidth (a return loss larger than $15 \mathrm{~dB}$ between $8.80-12.70 \mathrm{GHz}$ ) through capacitance compensation, which enlarges the dimension of the transition and increases the complexity of the printed launcher. An antipodal finline [12] consists in a curved geometry with a compact design, and the simulated results provide a return loss of around $20 \mathrm{~dB}$ in the W-band in the back-to-back transition. This configuration requires another transition between the microstrip and the launcher, as well as two shaped metallizations on both sides of the dielectric. A transition in the W-band between a microstrip patch and a full waveguide has been presented in [13]. This transition consists in a multistep geometry: an initial transition between a patch and a dielectrically filled reduced height waveguide (whose height is equal to the thickness of the microstrip substrate), a connection between the dielectrically filled waveguide and a standard WR10 through a tapered substrate, and a multi-section Chebyshev transformer with different heights. Such a transition covers the entire W-band with a return loss of more than $15 \mathrm{~dB}$. Finally, a design for the V-band with an impedance transformer and a direct short of the patch on the first ridge of the Chebyshev is presented in [14].

The aim of this work is the design of an in-line transition between a microstrip on an alumina substrate and a typical WR-22 waveguide (with dimensions of $5.69 \times 2.84 \mathrm{~mm}$ ) in the Q-band $(33-50 \mathrm{GHz})$ receiver of the Sardinia Radio Telescope. The transition is designed to be placed between the antenna and the module of down-conversion from the Q-band to the IF of 1-1.8 GHz. A high return loss is required over the entire operating band if the quality of the signal is not to be affected.

The launcher is built using microstrip technology and consists on a simple small patch matched to the waveguide through a ridged configuration, on which the patch is shorted, and a shaped back-short. A Chebyshev impedance transformer provides the matching from the ridge to the full waveguide, and it has been optimized through the PSO algorithm to balance the effects of the discontinuities. Such a geometry does not require any modification in the height of the waveguide, nor the application of antennas with complex geometry such as bow-tie or quasi-Yagi. This increases the ease of fabrication and the compactness of the project: the launcher is less than $1 \mathrm{~mm}$ long, whereas the impedance 
transformer is about $1 \mathrm{~cm}$. The performance of such a transition provides a high return loss $(>20 \mathrm{~dB})$ over the entire Q-band.

\section{Design}

\subsection{Theoretical Model}

The transition between the microstrip and the WR22 waveguide has to accomplish two different requirements: impedance matching and a modal conversion between the two devices. The modal conversion from the quasi-TEM mode in the microstrip to the $T E_{10}$ in the WR-22 requires a structure that is a trade-off between the devices in terms of field distribution. On the other hand, the matching between the low impedance of the microstrip and the high impedance of the waveguide is obtained using a section of ridged waveguide with stepped ridges.

The launcher is inserted from the back-short of the waveguide and lies on a plane parallel to the wide side of the waveguide, such that the E-planes of the two devices are parallel (Figure 1). The strip enters close to the upper wall, at a very small distance from that wall. The dielectric slab is below the strip (Figure 2, right), such that a very small air gap exists between the strip and the upper waveguide wall, and a large vertical electric field is produced in this air gap. The microstrip is centered along the wide side and placed with an offset along the small side, close to the metallic wall (in dark grey, Figure 2).

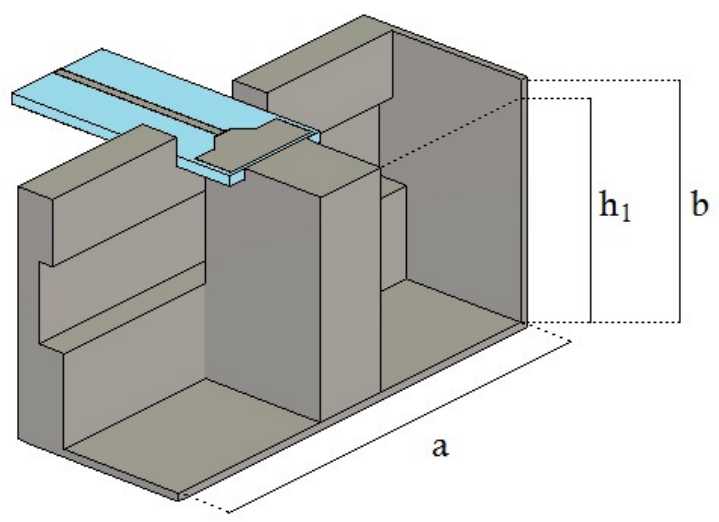

Figure 1. Geometry.
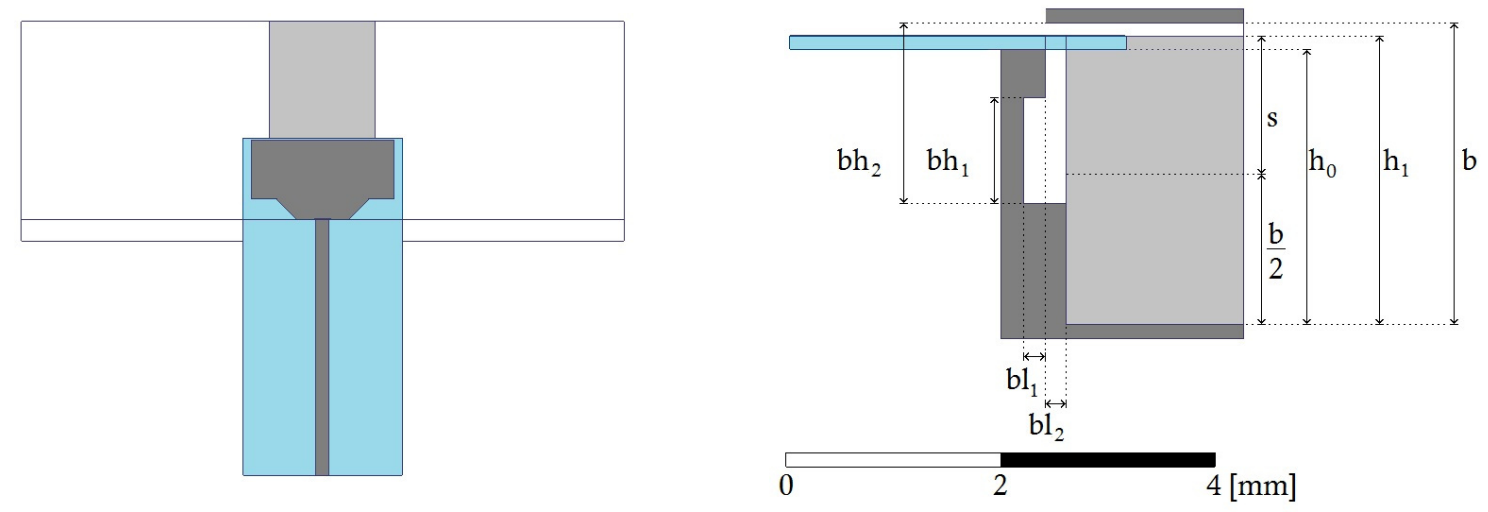

Figure 2. Geometry: top (left) and side (right) view. 
The waveguide transverse field configuration at the patch section fits the field in a ridged waveguide: it is concentrated in the center (the ridged zone) of the wide side, whereas it is weak in the rest of the transverse section; in this way, the transition couples the fields in the two devices. Figure 3 shows the field distribution on the transverse sections of the guide in correspondence of the patch (left) and the first ridge (right). The dielectric slab (shown in blue in Figure 2) is placed on a ridge (in light grey) centered along the long side of the waveguide. The final configuration is shown in Figure 4.
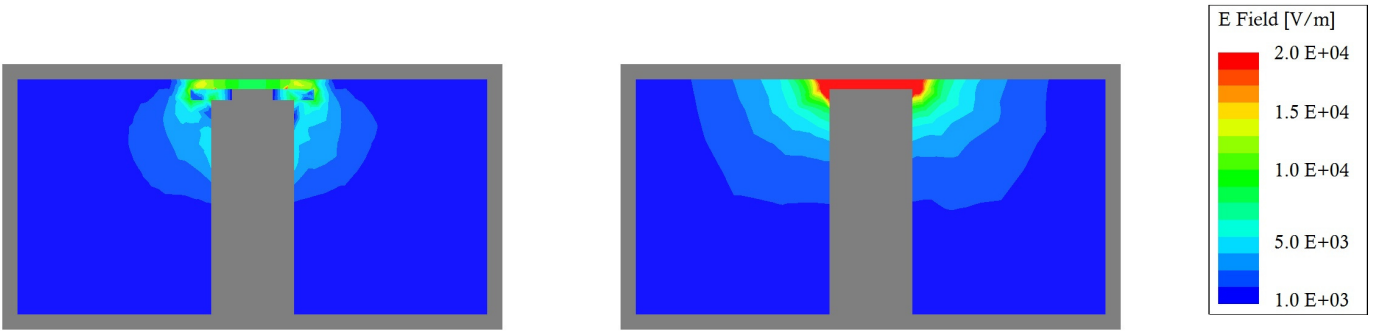

Figure 3. Field distribution in the end launcher step (left) and in the first ridge step (right). The metallic walls and the ridge are shown in grey.

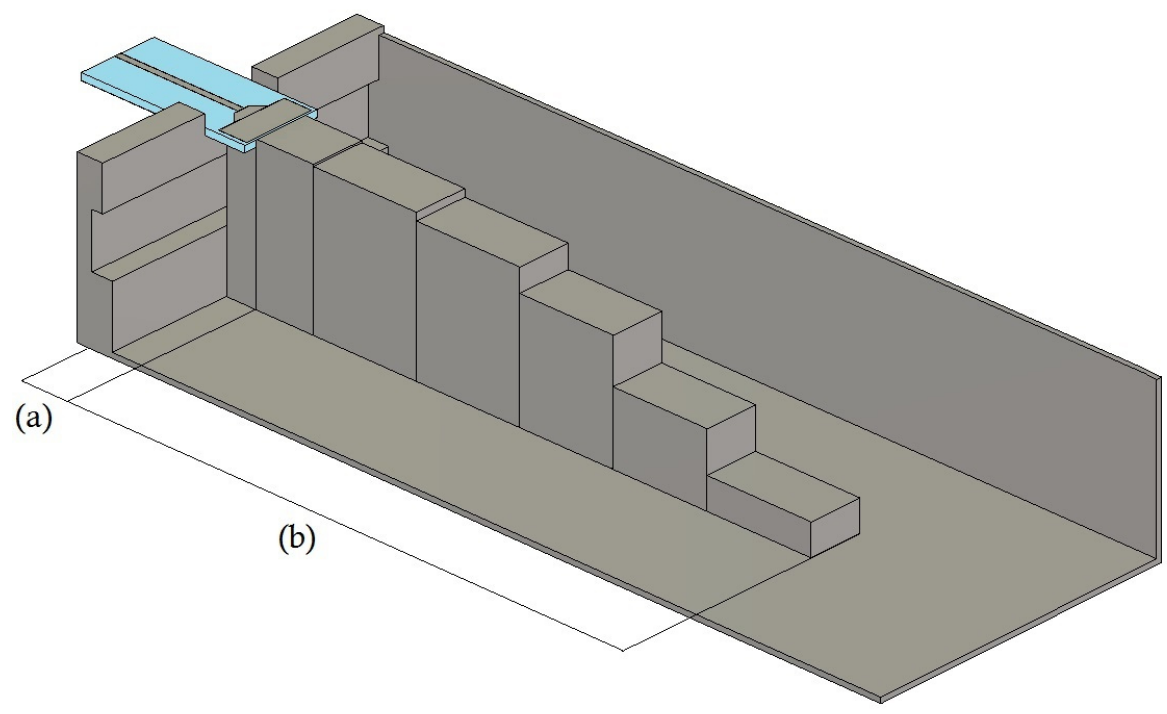

Figure 4. Final geometry, with launcher (a) and matching impedance (b) structures.

The size of the ridge is strongly related to the position of the end-launcher along the narrow side of the waveguide. The end-launcher is shorted on the ridge, so this is a crucial point to be taken into account. The height $h_{1}$ of the first ridge after the substrate is aligned with the offset of the patch and is slightly lower than the short side of the waveguide $b$. This step is then matched to the full waveguide through a ridge stepped transformer. Finally, the shape of the back-short of the waveguide is a further parameter used to reach the desired matching.

\subsection{Design}

The microstrip was built on an Alumina $98 \%$ substrate (thickness $0.127 \mathrm{~mm}, \epsilon_{r}=9.8, \tan \delta_{e}=0.006$ ). Such a substrate terminates inside the waveguide on the first ridge, whereas the ground metallization is present only in the part outside the guide. The waveguide is made of aluminum, and the strip metallization is copper. Figure 2 shows the geometry of the transition from the top and from a side. Figure 5 provides a focus on the launcher. 

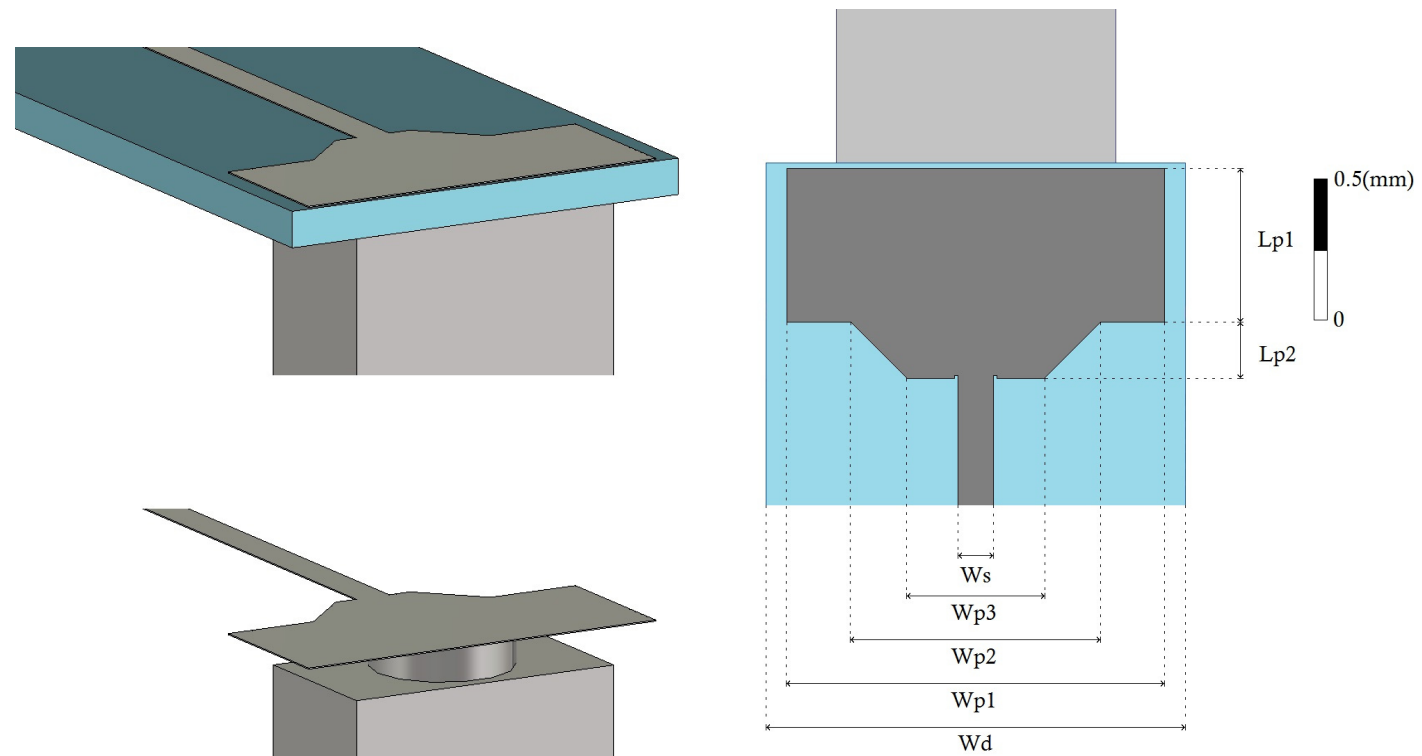

Figure 5. End launcher: via hole configuration with and without the substrate (left) and patch geometry (right).

The patch lies on a dielectric slab Wd wide. The offset of the microstrip with respect to the center of the guide is $s=1.3 \mathrm{~mm}$ (see Figure 2). The desired return loss is obtained by modeling appropriate matching elements in the microstrip and in the waveguide. The patch is matched to the $50 \Omega$ line by shaping its rear part and is shorted to the ridge through a via hole, as shown in Figure 5. The via hole connection provides a better ground short circuit compared to the short circuit realized with a two-component glue (like EPOXY H20E), which can affect a perfect contact. In particular, the via avoids the drawbacks due to the series resistance that the glue forms between the patch and the ridge. Furthermore, it avoids the undesired thickness variation caused by the glue deposition, which modifies the air gap between the strip and the waveguide wall. The relative size of the via hole diameter is sufficiently large $(0.5 \mathrm{~mm})$ to allow for easy operation, and this affects the size of the patch. The patch consists of a rectangular part with a size of $L p_{1} \times W p_{1}$ and a tapered part with widths of $W p_{2}$ and $W p_{3}$, and a length of $L p_{2}$, which matches the rectangular patch with the $50 \Omega$ line (Ws $=0.127 \mathrm{~mm}$ wide). The values of $L p_{1}$ and $W p_{1}$ are, respectively, $1.35 \mathrm{~mm}$ and $0.55 \mathrm{~mm}$, and they are strongly influenced by the via hole. This results in a larger size of the patch with respect to a standard radiating patch on such a substrate. The tapered part of the patch has dimensions of $W p_{2}=0.69 \mathrm{~mm}, W p_{3}=0.5 \mathrm{~mm}$, and $L p_{2}=0.2 \mathrm{~mm}$.

The impedance matching can be achieved by enlarging the short side of the guide in the first step, but the need for a compact structure leads to obtain the same results by modifying the shape of the back-short. Such a cavity acts as a tuning stub whose reactive behavior is used to improve the matching in the transition. Due to the complexity of the structure, and the coupling between the discontinuities and the patch, a parametric sweep has been applied to determine the optimal size of the back-short. The first step of the ridged section, in correspondence to the tapered section of the patch $(b l 2=l p 2)$, has a reduced height of $b h 2=1.7 \mathrm{~mm}$. Moreover, as shown in Figure 2, a shaped hollow has been applied behind this, with a size of $b l_{1}=0.2 \mathrm{~mm}$ and $b h_{1}=1 \mathrm{~mm}$, allowing for a two section "tuning stub." In this way, the transverse section of the device is not increased.

The matching between the ridge where the patch is shorted and the full waveguide requires an impedance transformer. Ideally, a tapered transformer provides the best solution. However, the request of a very compact transition results in a multi-step transformer. A solution that guarantees a compact geometry in as few steps as possible is the Chebyshev transformer [15]. 


\subsection{Optimization}

The Chebyshev transformer is described analytically in [16], and the theoretical values of the impedances can be easily obtained. The realization of such a transformer using ridge waveguides, however, is by no means straightforward. First of all, only approximate expressions are known for the (power-current) impedance of ridge waveguides [17]. Moreover, the behavior of discontinuities between ridge waveguides with different ridges in quantitative terms is even less known. However, since each discontinuity has a reactive effect, we expect that the length of each transformer section be different from the nominal $\lambda / 4$ and must be optimized during the design phase.

Typically, the devices involved in the receiving chain must have a return loss higher than $20 \mathrm{~dB}$. The requirement of a compact solution reflects the use of a small number of ridges; on the other side, the return loss improves by increasing the number of steps of the transformer. Therefore, in order to obtain such a return loss with a few ridged waveguide sections, the lengths of the sections and the heights of the ridges must be very accurate, and an automatic optimization procedure is needed. Thus, by imposing the same width for all the ridges, equal to $w=1 \mathrm{~mm}$, the optimization involves the heights (which modify the impedance value) and the lengths (which balance the reactive phenomena) of each ridge step by using suitable CAD software, which provides precise numerical solutions.

A five-step transformer was chosen, so 10 variables need to be optimized. For quicker convergence, such variables have been defined starting from the analytic values [17] evaluated at a central frequency of $41.5 \mathrm{GHz}$, and the optimization variables are the additional correction terms. Let $h_{t}^{*}, l_{t}^{*}$ be the height and length computed for a generic step, and the final values $h_{t}, l_{t}$ will be modified by the values $\Delta h, \Delta l$ to be optimized:

$$
h_{t}=h_{t}^{*}+\Delta h \quad l_{t}=l_{t}^{*}+\Delta l .
$$

The optimization is obtained by applying the PSO algorithm through a synergistic operation of Matlab and HFSS (Figure 6). The PSO iteratively manipulates the variables to be optimized according to some mathematical formula, to find the best solution of the problem in a determined solution space $[18,19]$. The main task of every optimization procedure is the evaluation of the objective function for the present values of the variables. In our case, the geometric parameters of the transformer, i.e., the optimization variables, computed at each step by the PSO are passed to HFSS. Its finite element solver then computes the return loss of this transformer configuration and, from it, the value of the objective function. The latter is simply the lowest return loss in the required bandwidth.

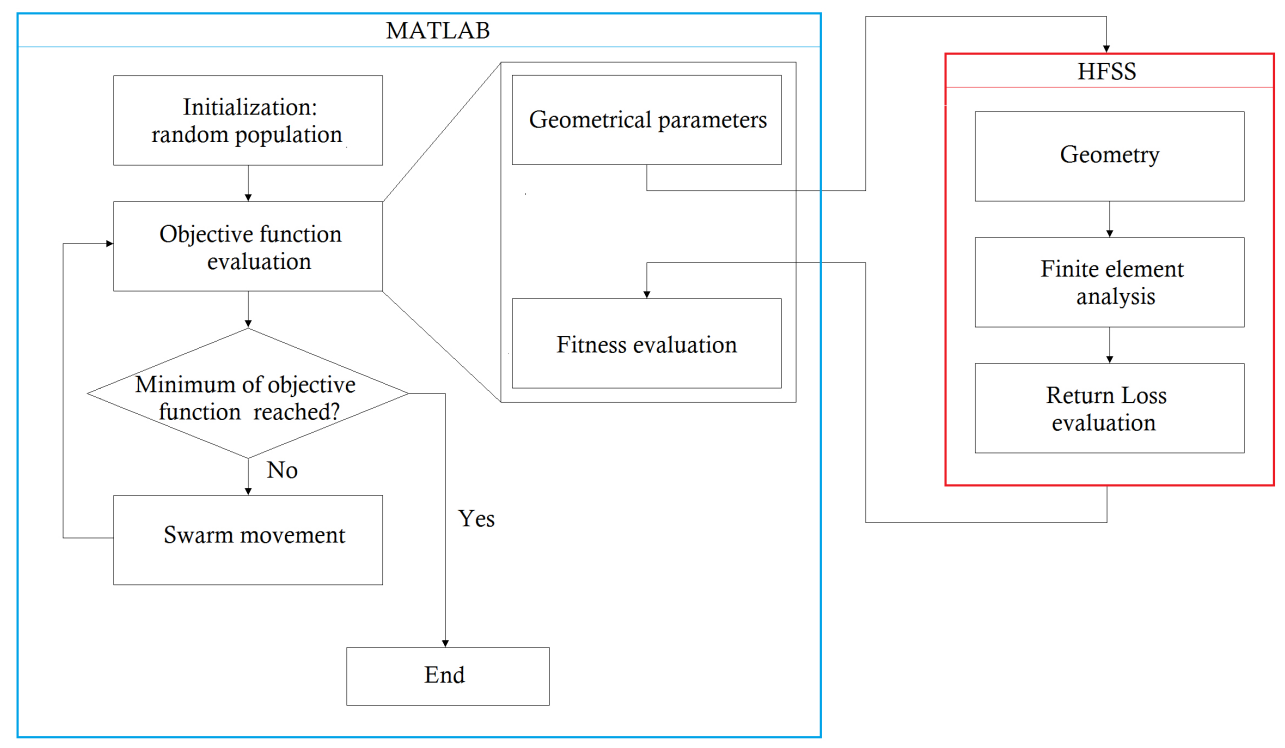

Figure 6. Optimization scheme: PSO and HFSS integration. 


\section{Results}

The final configuration of the transition is shown in Figure 4. The device was simulated using Ansys HFSS, a finite element method commercial software, particularly suited for the simulation and design of waveguide structures and antennas [20]. Very good agreement with experimental measurements [21] was found, even at millimeter wave frequencies [22-24]. The waveguide sections are modeled as aluminum, and the microstrip metallization modeled as copper. The transition is only $11.69 \mathrm{~mm}$ long (from the back-short of the waveguide to the last ridge), in agreement with the compactness requirement. By considering an indefinite length waveguide, the return loss is evaluated on a section of the microstrip and constitutes the objective function optimized by the PSO. Figure 7 shows a return loss greater than $24 \mathrm{~dB}$ over the entire Q-band, ensuring wideband matching. The insertion loss was measured between the same section and the full waveguide, beyond the impedance transformer. The insertion loss was lower than 0.26 , so a good power transmission was provided.

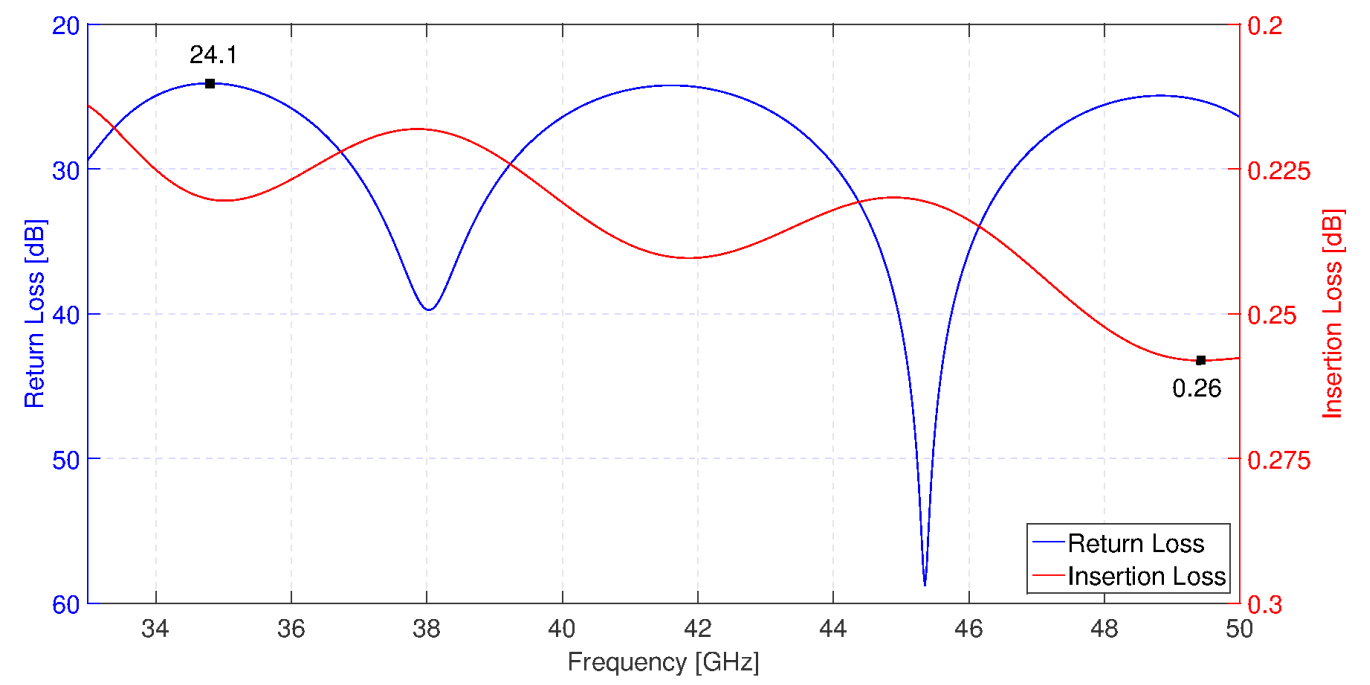

Figure 7. Simulated return loss and insertion loss (Ansys HFSS) of the transition.

Finally, Figure 8 shows the ridges that constitute the impedance transformer, whose optimized dimensions are listed in Table 1 . The first ridge touches the back-short of the waveguide and provides the short for the end launcher: the via hole (on the left in Figure 8) passes through the substrate and shorts the patch.

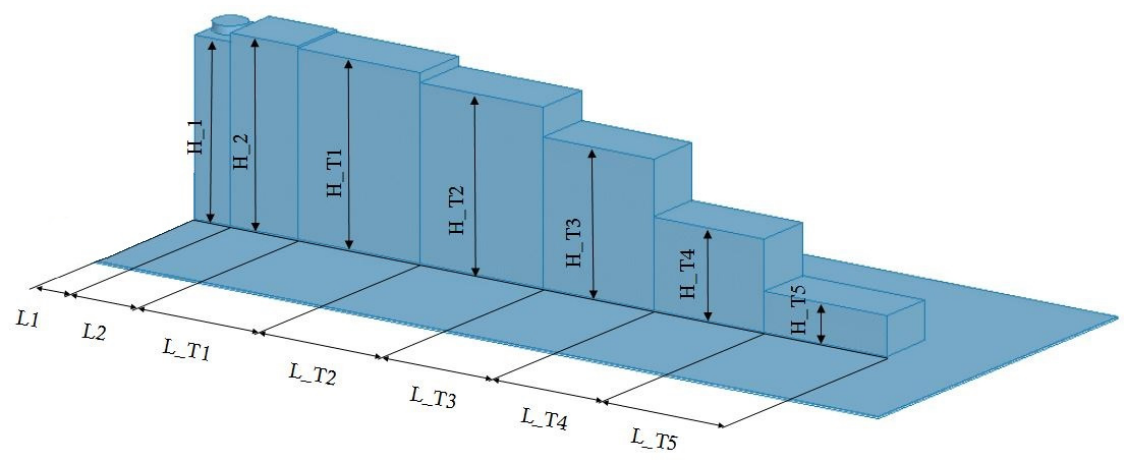

Figure 8. Size details of the ridged impedance transformer. 
Table 1. Geometrical parameters of the ridges.

\begin{tabular}{cccc}
\hline Height & [mm] & Length & [mm] \\
\hline H_1 & 2.6 & L_1 & 0.57 \\
H_2 & 2.72 & L_2 & 1.1 \\
H_T1 & 2.69 & L_T1 & 2 \\
H_T2 & 2.55 & L_T2 & 2 \\
H_T3 & 2.13 & L_T3 & 1.81 \\
H_T4 & 1.31 & L_T4 & 1.81 \\
H_T5 & 0.57 & L_T5 & 2 \\
\hline
\end{tabular}

\section{Conclusions}

In this paper, an approach to the design of an in-line waveguide-to-microstrip transition has been described. It consists in a via hole, which shorts the launcher on a ridge impedance transformer, and requires the modification of the waveguide back-short. The final geometry is simple in order to guarantee easy fabrication and integration, and provides a high return loss in the entire Q-band. It is scalable at lower, and possibly higher, frequencies, even if, especially in the latter case, further investigation into the mechanical tolerances of the fabrication process is required.

Author Contributions: All the authors contribute to the design of the component. Marco Simone wrote the paper. Conflicts of Interest: The authors declare no conflict of interest.

\section{References}

1. Yeomas, M.E. Radar Receivers. In Radar Handbook; Skolnik, M.I., Ed.; McGraw Hill: New York, NY, USA, 2008.

2. Kraus, J.D. Radio Astronomy Fundamentals. In Radio Astronomy, 2nd ed; Cygnus-Quasar Books: Powell, OH, USA, 1986; pp. 101-104.

3. Valente, G.; Montisci, G.; Pisanu, T.; Navarrini, A.; Marongiu, P.; Casula, G.A. A Compact L-Band Orthomode Transducer for Radio Astronomical Receivers at Cryogenic Temperature. IEEE Trans. Microw. Theory Tech. 2015, 63, 3218-3227.

4. Orfei, A.; Carbonaro, L.; Cattani, A.; Cremonini, A.; Cresci, L.; Fiocchi, F.; Maccaferri, A.; Maccaferri, G.; Mariotti, S.; Monari, J.; et al. A Multi-Feed Receiver in the 18 to $26.5 \mathrm{GHz}$ Band for Radio Astronomy. IEEE Antennas Propag. Mag. 2010, 52, 62-72.

5. Ladu, A.; Valente, G.; Montisci, G.; Mazzarella, G. A wideband quadruple-ridged horn antenna for the multifeed S-band receiver of the Sardinia Radio Telescope. J. Electromagn. Waves Appl. 2016, 30, 1207-1216.

6. Wheeler, G. Broadband waveguide-to-coax transitions. In Proceedings of the 1958 IRE International Convention Record, New York, NY, USA, 21-25 March 1966; Volume 5, pp. 182-185.

7. Yao, H.-W.; Abdelmonem, A.; Liang, J.-F.; Zaki, K.A. Analysis and design of Microstrip-to-Waveguide Transitions. IEEE Trans. Microw. Theory Tech. 1994, 42, 2371-2380.

8. Deslandes, D.; Wu, K. Integrated Microstrip and Rectangular Waveguide in Planar Form. IEEE Microw. Wirel. Comp. Lett. 2001, 11, 68-70.

9. Simeoni, M.; Coman, C.I.; Lager, I.E. Patch End-Launchers-A Family of Compact Colinear Coaxial-to-Rectangular Waveguide Transitions. IEEE Trans. Microw. Theory Tech. 2006, 54, 2371-2380.

10. Kaneda, N.; Qian, Y.; Itoh, T. A Broad-Band Microstrip-to-Waveguide Transition Using Quasi-Yagi Antenna. IEEE Trans. Microw. Theory Tech. 1999, 47, 2562-2567.

11. Fang, R.-Y.; Wang, C.-L. Miniaturized Microstrip-to-Waveguide Transition Using Capacitance-Compensated Broadside-Coupled Microstrip Line. IEEE Trans. Comp. Packag. Manuf. Technol. 2013, 3, 1588-1596.

12. Jing, S.; Fa-guo, L.; Li-hua, H.; Xiao-ying, S.; Yan-qiu, Z. Waveguide-to-Microstrip Antipodal Finline Transition at W Band. In Proceedings of the 2013 Third International Conference on Instrumentation, Measurement, Computer, Communication and Control, Shenyang, China, 21-23 September 2013; pp. 510-513. 
13. Rebollo, A.; Larumbe-Gonzalo, B.; Gonzalo, R.; Ederra, I. Full W-Band Microstrip-to-Waveguide Inline Transition. In Proceedings of the 8th European Conference on Antennas and Propagation (EuCAP 2014), The Hague, The Netherlands, 6-11 April 2014; pp. 2591-2593.

14. Han, K.Y.; Pao, C.-K. A V-band waveguide to microstrip inline transition. In Proceedings of the 2012 IEEE/MTT-S International Microwave Symposium Digest, Montreal, QC, Canada, 17-22 June 2012.

15. Collin, R.E. Theory and Design of Wide-Band Multisection Quarter-Wave Transformers. Proc. IRE 1955, 43, $179-185$.

16. Pozar, D.M. Impedance Matching and Tuning. In Microwave Engineering; Pozar, D.M., Ed.; John Wiley \& Sons, Inc.: Hoboken, NJ, USA, 2011; pp. 256-261.

17. Helszajn, J.; Caplin, M. Impedance and propagation in ridge waveguides using the transverse resonance method. In Ridge Waveguides and Passive Microwave Components; Helszajn, J., Ed.; IET Electromagnetic Waves Series; The Institution of Engineering and Technology: London, UK, 2000; pp. 46-49.

18. Simone, M.; Fanti, A.; Mazzarella, G. Ridge waveguide optimization with PSO algorithm. J. Electromagn. Waves Appl. 2015, 29, 199-209.

19. Simone, M.; Fanti, A.; Montisci, G.; Casula, G.A.; Mazzarella, G. Combined PSO-FDFD Optimization of Rectangular Ridged Waveguides. ACES J. 2016, 31, 144-151, ISSN 1054-4887.

20. Casula, G.A.; Mazzarella, G.; Montisci, G. Design of Shaped Beam Planar Arrays of Waveguide Longitudinal Slots. Int. J. Antennas Propag. 2013, doi:10.1155/2013/767342.

21. Yang, H.; Jin, Z.; Montisci, G.; Liu, Y.; He, X.; Casula, G.A.; Mazzarella, G. Design Equations for Cylindrically Conformal Arrays of Longitudinal Slots. IEEE Trans. Antennas Propag. 2016, 64, 80-88.

22. Tekkouk, K.; Hirokawa, J.; Sauleau, R.; Ando, M. Wideband and Large Coverage Continuous Beam Steering Antenna in the 60-GHz Band. IEEE Trans. Antennas Propag. 2017, 65, 4418-4426.

23. Hsu, S.; Wei, K.-C.; Hsu, C.-Y.; Ru-Chuang, H. A 60-GHz Millimeter-Wave CPW-Fed Yagi Antenna Fabricated by Using 0.18- $\mu \mathrm{m}$ CMOS Technology. IEEE Electron Device Lett. 2008, 29, 625-627.

24. Mikulasek, T.; Lacik, J.; Puskely, J.; Raida, Z. Design of aperture-coupled microstrip patch antenna array fed by SIW for $60 \mathrm{GHz}$ band. IET Microw. Antennas Propag. 2016, 10, 288-292.

(C) 2018 by the authors. Licensee MDPI, Basel, Switzerland. This article is an open access article distributed under the terms and conditions of the Creative Commons Attribution (CC BY) license (http:/ / creativecommons.org/licenses/by/4.0/). 